\title{
Up-regulation of Foxo4 mediated by hepatitis B virus X protein confers resistance to oxidative stress-induced cell death
}

\author{
RATAKORN SRISUTTEE ${ }^{1}$, SANG SEOK KOH ${ }^{3}$, EUN HEE PARK ${ }^{1}$, IL-RAE CHO $^{1}$, HYE JIN MIN $^{3}$, \\ BYUNG HAK JHUN ${ }^{2}$, DAE-YEUL YU ${ }^{4}$, SUN PARK ${ }^{5}$, DO YUN PARK ${ }^{6}$, MI OCK LEE ${ }^{7}$, \\ DIEGO H. CASTRILLON ${ }^{8}$, RANDAL N. JOHNSTON ${ }^{9}$ and YOUNG-HWA CHUNG ${ }^{1}$ \\ ${ }^{1}$ WCU Department of Cogno-Mechatronics Engineering and ${ }^{2}$ Department of Nanomedical Engineering, BK21 Nanofusion \\ Technology Team, Pusan National University, Busan; ${ }^{3}$ Therapeutic Antibody Research Center and ${ }^{4}$ Disease Model \\ Research Center, Korea Research Institute of Bioscience and Biotechnology, Daejeon; ${ }^{5}$ Department of Microbiology, \\ Ajou University School of Medicine, Suwon; ${ }^{6}$ Department of Pathology, College of Medicine, Pusan National \\ University, Yangsan; ${ }^{7}$ College of Pharmacy, Seoul National University, Seoul, Republic of Korea; \\ ${ }^{8}$ Department of Pathology and Simmons Comprehensive Cancer Center, University of Texas \\ Southwestern Medical Center, Dallas, TX, USA; ${ }^{9}$ Department of Biochemistry and \\ Molecular Biology, University of Calgary, Calgary, Alberta, Canada
}

Received January 27, 2011; Accepted March 17, 2011

DOI: $10.3892 /$ ijmm.2011.699

\begin{abstract}
The hepatitis B virus X (HBX) protein, a regulatory protein of the hepatitis $B$ virus (HBV), has been shown to generate reactive oxygen species (ROS) in human liver cell lines; however, the mechanism by which cells protect themselves under this oxidative stress is poorly understood. Here, we show that HBX induces the up-regulation of Forkhead box class O 4 (Foxo4) not only in Chang cells stably expressing HBX (Chang-HBX) but also in primary hepatic tissues from HBX-transgenic mice. HBX also increased ROS, but reduction of the abundance of ROS using $\mathrm{N}$-acetylcystein (NAC) diminished the levels of Foxo4. Elevated Foxo4 was also detected in nuclei of Chang-HBX cells but not in Chang cells stably expressing the vector (Chang-Vec), suggesting that HBX activates the transcriptional activity of Foxo4. When we examined whether HBX bypasses JNK signaling that targets Foxo4, we found that the activity of JNK but not of ERK is required for the up-regulation of Foxo4 even in the presence of HBX. Furthermore, the reduction of Foxo4 levels using siRNA or a JNK inhibitor rendered Chang-HBX cells sensitive to apoptosis under oxidative stress, suggesting that up-regulation of Foxo4 mediated by HBX enhances resistances to oxidative stress-induced cell death. Accordingly, we propose that Foxo4
\end{abstract}

Correspondence to: Dr Young-Hwa Chung, WCU Department of Cogno-Mechatronics Engineering, Pusan National University, Busan 609-732, Republic of Korea

E-mail: younghc@pusan.ac.kr

Key words: Forkhead box class O 4, hepatitis B virus X, JNK, reactive oxygen species, oxidative stress may be a useful target for suppression in the treatment of $\mathrm{HBV}$-associated hepatocellular carcinoma cells.

\section{Introduction}

The human hepatitis B virus (HBV) induces acute and chronic hepatitis and is closely associated with the incidence of human liver cancer $(1,2)$. Among the four proteins that are derived from the HBV genome, the hepatitis B virus X (HBX) protein is involved in multiple signaling pathways associated with cell survival and proliferation. Cell signal transduction pathways that are activated by HBX include the Jak1/Stat3, PI-3 kinase pathways (3-6) and the Ras/Raf/MAPK signaling cascade which leads to $\mathrm{NF}-\kappa \mathrm{B}$ activation $(7,8)$. In addition, HBX expression increases reactive oxygen species (ROS) via calcium signaling and cellular kinases, which results in the activation of the transcription factors $N F-\kappa B$ and Stat3 (3). Further studies have revealed that HBV-induced oxidative stress also stimulates the translocation of Raf-1. Src inhibitors or a dominant negative PAK mutant abolishes Raf-1 mitochondrial translocation mediated by $\operatorname{HBX}(9)$.

The Forkhead box class O (Foxo) subfamily proteins consist of the functionally related proteins, Foxo1, Foxo3a, Foxo4 and Foxo6 (10). Growth factors negatively regulate Foxo transcriptional activity by phosphorylation-mediated nuclear exclusion $(11,12)$ through Akt kinase signaling. This pathway is evolutionarily conserved from Caenorhabditis elegans to humans. However, nutrient deficiency or oxidative stress induce Foxo activity, which respectively activate the gene expression of pyruvate dehydrogenase kinase 4 and of $\mathrm{Mn}$ superoxide dismutase (SOD) (13-15). This seems to suggest that Foxo plays a protective role against stress and is vital for cell survival. Conversely, there is evidence to show that increased Foxo activity induces apoptosis through a Foxomediated regulation of various pro-apoptotic genes, including 
Fas ligand, and Bim $(11,16,17)$. Sustained inhibition of Foxo activity is emerging as a critical event in tumor cells, suggesting that Foxo may act as a tumor suppressor (18). Recent studies have also reported that Foxo is involved in the up-regulation of multi-drug resistance in leukemia cells (19).

The goal of the present study was to explore the pathways linking HBX expression with the protection of cells under oxidative stress. We herein report that the Foxo4 transcriptional factor plays a crucial role in protecting a human liver cell line from oxidative stress-induced cell death. On the basis of this result, we propose that suppression of Foxo4 may provide an effective therapeutic regimen for treatment of HBV-associated hepatocellular carcinoma cells.

\section{Materials and methods}

Cell cultures and transgenic mice. Parental Chang cells and Chang cells stably expressing vector (Chang-Vec) or HBX (Chang-HBX) were cultured in DMEM supplemented with $10 \%$ FBS and $1 \%$ penicillin and streptomycin. HBX transgenic mice (C57BL/6) described previously (20) were maintained under an SPF barrier area in the Korea Research Institute of Bioscience and Biotechnology (KRIBB). The HBX transgenic mice were sacrificed at 24 weeks after birth and age-matched non-transgenic mice (C57BL/6) were used as a negative control. Animal care and procedures were performed in compliance with the policy of the KRIBB Animal Care and Use Committee.

Reagent and antibodies. PD98059, SP600125 and SB202190 were used to inhibit the activation of MAPK including ERK, JNK and p38, respectively, and were added to cells at different concentrations for $12 \mathrm{~h}$. N-acetylcysteine (NAC) was used to reduce ROS concentration, and PP2 and wortmannin were used to inhibit Src and PI3 kinase activity, respectively. All inhibitors were purchased from Calbiochem (San Diego, CA) For immunoblotting, anti-Foxo4, MnSOD and $\beta$-tubulin antibodies were obtained from Santa Cruz Biotechnology (Santa Cruz, CA). Akt, its phospho-specific antibody (Ser-705), and anti-PARP antibody were purchased from Cell Signaling (Danvers, MA).

siRNA transfection. Cells were trypsinized and incubated overnight to achieve $60-70 \%$ confluency before siRNA transfection. Foxo4 siRNA [60 nM, 5'-UCUCACCUCUUCCCAU UCC(dTdT)-3' as sense and 5'-GGAAUGGGAAGAGGUG AGA(dTdT)-3' as antisense] (Bioneer Corp., Korea) described elsewhere (21) or control siRNA (22) were mixed with Lipofectamine 2000 (Invitrogen). The cells were incubated with the transfection mixture for $6 \mathrm{~h}$ and then rinsed with DMEM containing $10 \%$ serum. The cells were incubated for $48 \mathrm{~h}$ before harvesting.

Western blot assays. Cells were harvested and lysed with lysis buffer [150 mM NaCl, $1 \%$ NP-40, $50 \mathrm{mM}$ Tris-HCl (pH 7.5)] containing $0.1 \mathrm{mM} \mathrm{Na}_{2} \mathrm{VO}_{3}, 1 \mathrm{mM} \mathrm{NaF}$ and protease inhibitors (Sigma, St. Louis, MO). For immunoblotting, proteins from whole cell lysates were resolved by 10 or $12 \%$ SDS-PAGE and then transferred to nitrocellulose membranes. Primary antibodies were used at 1:1,000 or 1:2,000 dilutions, and secondary antibodies conjugated with horseradish peroxidase were used at 1:2,000 dilutions in 5\% nonfat dry milk. After final washing, nitrocellulose membranes were exposed for enhanced chemiluminescence assays using the LAS 3000 (Fuji, Japan).

Cellular fractionation. Cells cultured in $60 \mathrm{~mm}$ plates were harvested with ice-cold PBS and cell pellets were lysed with $400 \mu 1$ of TTN buffer [20 mM Tris- $\mathrm{HCl}$ (pH 7.4), $0.05 \%$ Triton X-100, $150 \mathrm{mM} \mathrm{NaCl}, 1 \mathrm{mM}$ EDTA, 1 mM DTT, 10\% glycerol, $0.5 \mathrm{mM}$ PMSF, and $1 \mathrm{X}$ cocktail protease inhibitor] on ice for $20 \mathrm{~min}$ followed by centrifugation at $10,000 \mathrm{x}$ g for $15 \mathrm{~min}$. The supernatants served as the cytosol fractions, and the pellets as the insoluble fractions which were subsequently solubilized in $400 \mu 1$ of RIPA buffer [ $50 \mathrm{mM}$ Tris- $\mathrm{HCl}$ (pH 7.4), $150 \mathrm{mM} \mathrm{NaCl}, 1$ mM EDTA, 1 mM DTT, $1 \%$ NP-40, $0.5 \%$ deoxycholic acid, $0.1 \%$ SDS, $10 \%$ glycerol, $0.5 \mathrm{mM}$ PMSF, and $1 \mathrm{X}$ cocktail protease inhibitor] on ice for $30 \mathrm{~min}$ and were centrifuged at $12,000 \mathrm{xg}$ for $15 \mathrm{~min}$. Thereafter, the supernatants were used as the nuclear extracts.

Measurement of ROS. As described elsewhere (23), intracellular ROS levels were determined by using an oxidative-sensitive fluorescence dye, 2',7'-dichlorodihydrofluorescein diacetate (DCF, Molecular Probes, Eugene, OR). Cells were treated with $20 \mu \mathrm{M}$ DCF for $30 \mathrm{~min}$, harvested after addition of $0.25 \%$ trypsin-EDTA, and extensively washed with PBS. The fluorescence intensities of the cells were measured at $485 \mathrm{~nm}$ for excitation and at $530 \mathrm{~nm}$ for emission (Victor3, Perkin-Elmer, MA).

Immunofluorescence. Cells were fixed with $4 \%$ paraformaldehyde for $15 \mathrm{~min}$, permeabilized with cold acetone for 15 min, blocked with $10 \%$ goat serum for $30 \mathrm{~min}$, and reacted with a 1:100 dilution of the primary antibody for $30 \mathrm{~min}$ at room temperature. After incubation, the cells were extensively washed with PBS, incubated with a 1:500-diluted Alexa Fluor 680-conjugated rabbit anti-goat IgG antibody (Molecular Probes) in PBS for $30 \mathrm{~min}$ at room temperature and washed three times with PBS. For nuclear staining, a Hoechst 33258 staining solution $(4 \mu \mathrm{g} / \mathrm{ml})$ (Sigma) was added for $30 \mathrm{~min}$. The stained cells were mounted with PBS containing $10 \%$ glycerol and photographed with a confocal microscope (LSM 510, Zeiss, Germany).

Statistical analysis. ROS measurement and cell proliferation assay were performed in triplicate. Data are represented as the means + standard deviations. The Student's t-test was used for the statistical analyses, with $\mathrm{P}<0.05$ defined as significant.

\section{Results and Discussion}

HBX expression induces intracellular oxidative stress, leading to up-regulation of Foxo4 protein level. Since expression of HBX, a regulatory HBV protein generates ROS in human liver cell lines (24), we wished to determine how these cells are able to protect themselves from this induced oxidative stress. We found that Chang-HBX produce more ROS than Chang-Vec (Fig. 1A), consistent with a previous study (24). Since the Foxo4 transcription factor is known to be involved in increasing cell survival under oxidative stress (13), 
A

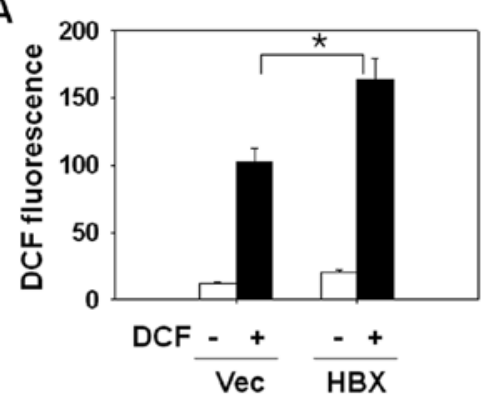

D

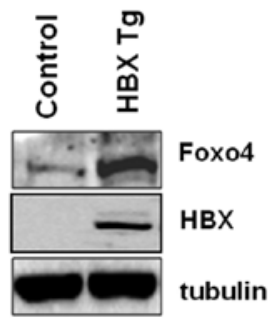

B

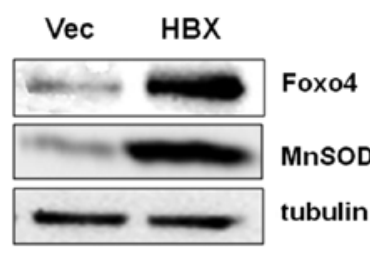

$\mathrm{E}$

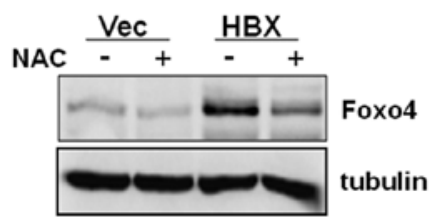

C

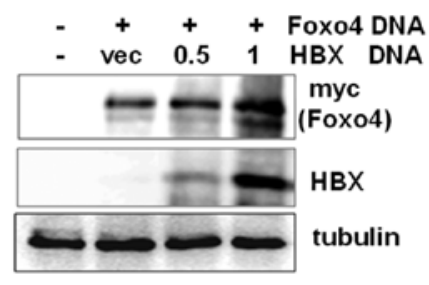

$\mathbf{F}$

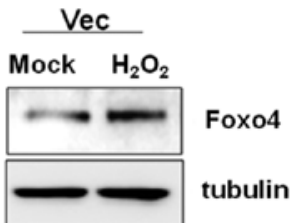

Figure 1. HBX-mediated oxidative stress induces up-regulation of Foxo4. (A) Chang-Vec and Chang-HBX cells were treated with 2',7'-dichlorodihydrofluorescin diacetate (DCF) fluorescence dye $(20 \mu \mathrm{M})$ for $30 \mathrm{~min}$ and harvested for measurement of DCF fluorescence dye at $485 \mathrm{~nm}$ for excitation and at $530 \mathrm{~nm}$ for emission, after extensive washings. Error bars indicate standard deviation. "P<0.05. (B) The expression of Foxo4, MnSOD and tubulin in Chang-Vec and Chang-HBX cells were examined by immunoblotting using the corresponding antibody. (C) Chang cells were transfected with HBX expression vector $(0.5$ or $1 \mu \mathrm{g})$ or empty vector as a control in the presence of c-Myc tagged Foxo4 vector $(0.5 \mu \mathrm{g})$. At $48 \mathrm{~h}$ post-transfection, the expression of Foxo and HBX were examined by immunoblotting using anti-myc and anti-HBX antibodies, respectively. (D) The cell lysates of hepatic tissues from control (24-week-old, C57BL6, $n=2$ ) and HBX-transgenic mice (24-week-old, C57BL6, $n=2)$ were prepared. After proteins from the cell lysates were separated on 10 or $15 \%$ SDS-PAGE, Foxo4, HBX and tubulin were detected by immunoblotting using the corresponding antibody. (E) Chang-Vec and Chang-HBX cells were treated with $\mathrm{N}$-acetylcystein (NAC; $20 \mathrm{mM}$ ) for $12 \mathrm{~h}$ and harvested for immunoblotting to detect the level of Foxo4. (F) Chang-Vec cells were treated with $\mathrm{H}_{2} \mathrm{O}_{2}$ and harvested for immunoblotting to detect the level of Foxo4.

the protein levels of Foxo4 in Chang-HBX and Chang-Vec cells were compared. The stable expression of HBX induced a large increase in the levels of Foxo4 compared to that of Chang-Vec cells (Fig. 1B). We also observed an up-regulation of MnSOD, which was correlated with the enhanced level of Foxo4 (Fig. 1B). Furthermore, when we examined the Foxo4 level in HBX-expressing transgenic mice, Foxo proteins were found more abundantly in the primary hepatic tissues of HBX-transgenic mice compared to the control mice (Fig. 1D). Conversely, when we reduced endogenous ROS with NAC, we found that the level of Foxo4 decreased in Chang-Vec and especially Chang-HBX cells relative to the untreated control cells (Fig. 1E). This result suggests that the level of endogenous ROS dictates the abundance of Foxo4. To address directly whether ROS plays a key role in the up-regulation of Foxo4, we added $\mathrm{H}_{2} \mathrm{O}_{2}$ to Chang-Vec cells and found that $\mathrm{H}_{2} \mathrm{O}_{2}$ induced the expression of Foxo4 (Fig. 1F). Taken together, these results support the idea that the elevated ROS induced by HBX up-regulates the expression of Foxo4.

$H B X$ expression induces translocation of Foxo 4 into the nucleus. Moreover, when we examined the localization of Foxo4, a significant fraction of the over-expressed Foxo4 protein was found in the nuclear fraction in Chang-HBX cells, similar to the nuclear-cytoplasmic ratio of Foxo4 seen in Chang-Vec cells despite the low level of Foxo4 in these cells (Fig. 2A). We confirmed that the Foxo4 protein was partially translocated into the nuclei of Chang-HBX cells using a confocal microscopy (Fig. 2B). The Foxo4 protein was barely detectable in the cytoplasm of Chang-Vec cells, while
A

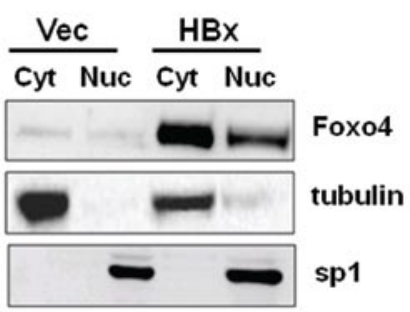

B
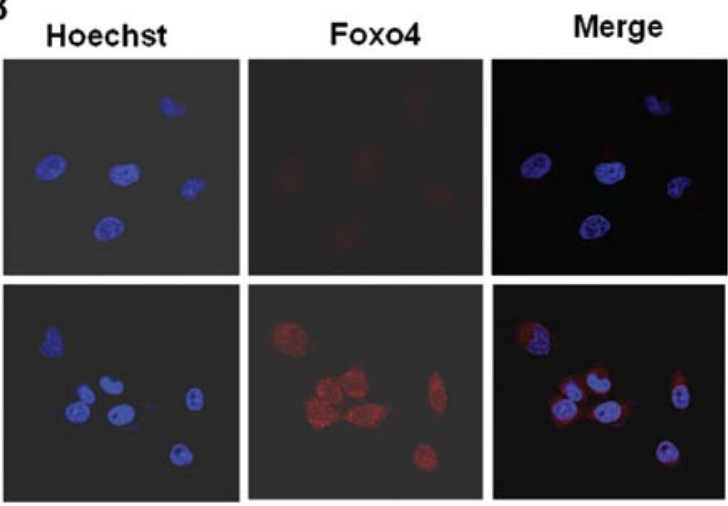

Vec
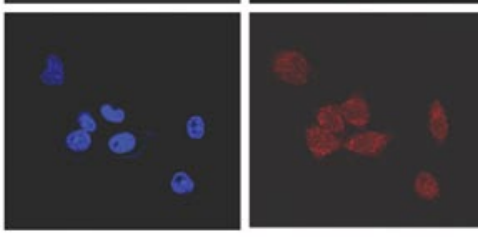

HBX

Figure 2. Presence of HBX induces translocation of Foxo4 into the nucleus (A) The cytosolic and nuclear fractions from Chang-Vec and Chang-HBX cells were separated and the level of Foxo4 was examined by immunoblotting. The cytosol and nuclear fraction were confirmed with tubulin and sp-1, respectively. (B) Chang-Vec and Chang-HBX cells were incubated on the chamber slide followed by fixation and permeabilization. The cells were stained with a goat anti-Foxo4 antibody followed by an Alexa Fluor 680-conjugated rabbit anti-goat IgG antibody. For nuclear staining, Hoechst staining solution was added before glycerol mounting. Immunofluorescence was detected with a Zeiss confocal microscope. 


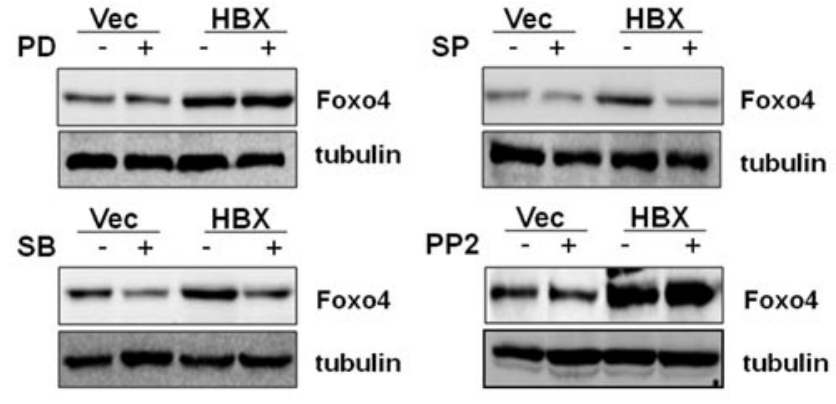

Figure 3. JNK activity is required for up-regulation of Foxo4 during oxidative stress even in the presence of HBX. Chang-Vec and Chang-HBX cells were treated with PD98059 (PD; $30 \mu \mathrm{M}$ ), SP600125 (SP; $20 \mu \mathrm{M}$ ), SB202190 (SB; $20 \mu \mathrm{M})$, or PP2 $(20 \mu \mathrm{M})$ inhibitors and harvested at $24 \mathrm{~h}$ post-infection. Foxo 4 and tubulin were examined by immunoblotting using the respective antibodies.

the Foxo4 protein was clearly detected in the nucleus as well as the cytoplasm of Chang-HBX cells. These results suggest that HBX expression induces a large up-regulation of Foxo4, accompanied by the translocation of Foxo4 into the nucleus where it is then available for transcriptional activity.

JNK inhibition sensitizes ROS-mediated cell death by downregulation of Foxo4. Although it has been reported that the activation of Foxo4 is dependent on JNK (25), we wanted to determine whether HBX expression provides an alternative signaling pathway that is independent of JNK activity for up-regulation of Foxo4. To test this hypothesis, we treated Chang-Vec and Chang-HBX cells with SP600125, a JNK inhibitor. In addition, we separately treated the cells with PD98059 or SB202190 to examine whether other MAPK kinases (ERK or p38 MAPK) are required for the up-regulation of Foxo4 even in the presence of HBX. The MAPK kinase inhibitors suppressed phosphorylation of their specific kinases (data not shown). The inhibition of ERK did not affect Foxo4 expression levels in Chang-Vec or Chang-HBX cells, while inhibition of JNK and p38 MAPK, which are both involved in stress signaling pathways, reduced Foxo4 expression in both cell lines (Fig. 3). These results suggest that up-regulation of Foxo4 requires JNK or p38 MAPK activity even in the presence of HBX. Since it has been shown that activated Raf-1 is involved in translocation of HBX into the mitochondria during oxidative stress (9), Chang-HBX cells were treated with PP2, a Src inhibitor, to block Raf-1 activation. Although blockage of Raf-1 activation was observed in the presence of this Src inhibitor (data not shown) (9) this administration was not accompanied by a reduction in Foxo4 levels (Fig. 3), suggesting that Foxo4 expression is not directly dependent on Raf-1 activity. On the other hand, multiple studies have argued that JNK is associated with cell proliferation. This is supported by the fact that higher activities of JNK have been associated with hepatocyte growth and regeneration (26). Furthermore, deletion of MKK4, an upstream kinase of JNK, has been shown to reduce JNK activity and to impair liver development in mice (27). Therefore, the biological role of JNK was examined in our model by treating Chang-HBX cells with the JNK inhibitor SP600125, and subsequently adding $\mathrm{H}_{2} \mathrm{O}_{2}$ to the cells. The Chang-HBX cells treated with SP600125 displayed reduced cell density and evidence of apoptotic cell death (cleaved PARP) under oxidative stress compared to $\mathrm{H}_{2} \mathrm{O}_{2}$-treated or mock-treated Chang-HBX cells (Fig. 4). In contrast, ERK inhibition did not enhance the sensitivity to oxidative stress in Chang-HBX cells (Fig. 4). These results confirmed that the up-regulation of Foxo4 by HBX-mediated JNK activation protects the cells from oxidative stress-induced apoptosis. Therefore, we identified a novel protective role of JNK in hepatic cell survival via Foxo4 under oxidative stress.
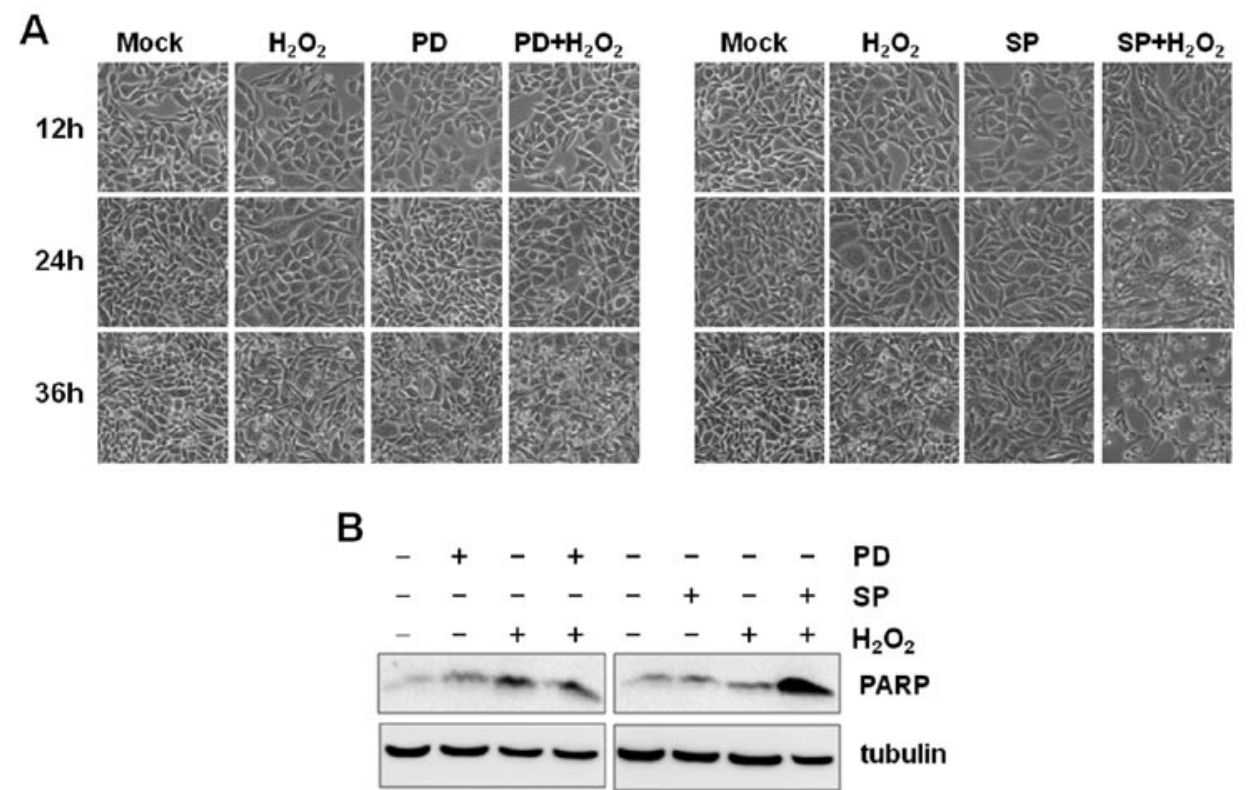

Figure 4. JNK activity is required for protection of Chang-HBX cells from oxidative stress-induced apoptosis. Chang-HBX cells were treated with PD98059 $(\mathrm{PD} ; 30 \mu \mathrm{M})$ or SP600125 (SP; $20 \mu \mathrm{M})$ for $12 \mathrm{~h}$ and followed by treatment with $\mathrm{H}_{2} \mathrm{O}_{2}$ for $36 \mathrm{~h}$. Cells were observed by microscopy at 12 , 24 and $36 \mathrm{~h}$ after $\mathrm{H}_{2} \mathrm{O}_{2}$ administration. The cells were harvested at $36 \mathrm{~h}$ post-treatment and apoptosis was detected by cleavage of PARP, a substrate of caspase 3 and 7. 
A
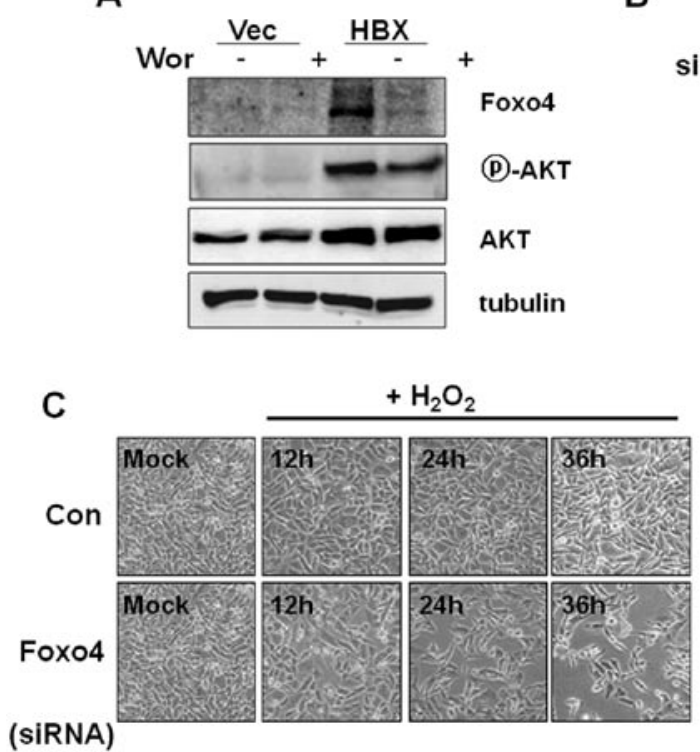

B
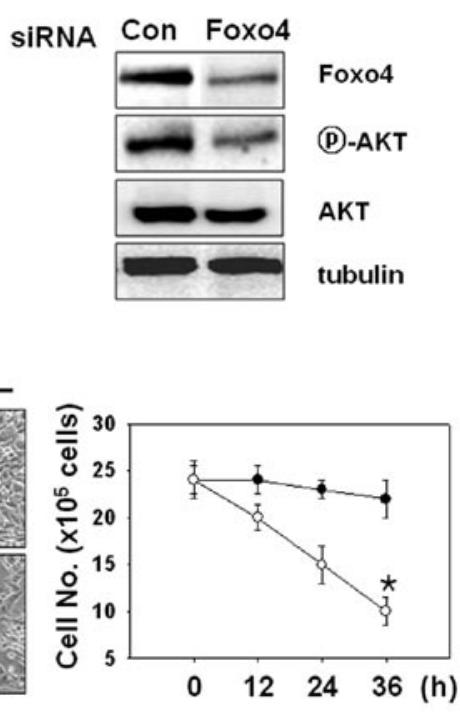

Figure 5. Suppression of Foxo4 sensitizes Chang-HBX cells to oxidative stress-induced cell death. (A) Chang-Vec and Chang-HBX cells were treated with wortmannin $(1 \mu \mathrm{M})$ to inhibit PI-3 kinase/Akt signaling and harvested at $24 \mathrm{~h}$ post-infection. The levels of Foxo4, phosphor-Akt, Akt and tubulin were examined by immunoblotting using the corresponding antibody. (B) Chang-HBX cells were transfected with Foxo siRNA (100 pmol) or control siRNA $(100 \mathrm{pmol})$ and harvested at $48 \mathrm{~h}$ post-transfection. Foxo4, phosphor-Akt, Akt and tubulin were detected by immunoblotting using the corresponding antibody. (C) Chang-HBX cells were transfected with Foxo4 siRNA or control siRNA for $12 \mathrm{~h}$, followed by treatment with $\mathrm{H}_{2} \mathrm{O}_{2}$ for $36 \mathrm{~h}$. Cells were observed by microscopy and counted using Trypan Blue staining at 12, 24 and $36 \mathrm{~h}$ after $\mathrm{H}_{2} \mathrm{O}_{2}$ administration. Open and closed circle indicate the viable cell number of Chang-HBX cells treated with Foxo4 siRNA and control siRNA, respectively. Error bars indicate standard deviation. ${ }^{*} \mathrm{P}<0.01$.

Suppression of Foxo4 sensitizes the resistance of Chang-HBX cells to oxidative stress-induced cell death. It has previously been reported that HBX expression can activate PI-3 kinase/ Akt-dependent signaling (28). Furthermore, Akt-mediated phosphorylation of Foxo has been shown in some systems to inhibit Foxo functions by promoting its interaction with 14-3-3 proteins and its relocalization from the nucleus to the cytosol (29), thereby leading to its proteasomal degradation through polyubiquitination by E3 ubiquitin ligase Skp2 (30). However, this proposed pathway of Akt-dependent degradation of Foxo4 is inconsistent with our observation of Foxo4 up-regulation, which evidently occurred even despite HBX-mediated constitutive activation of Akt. To resolve this contradiction, we first introduced wortmannin to inhibit PI-3 kinase/Akt signaling. Contrary to the earlier models, we found that Akt inhibition actually down-regulated Foxo4 levels compared to the mock-treated Chang-HBX cells (Fig. 5A), while a significant change in Foxo4 levels could not be observed in Chang-Vec cells due to the low expression of Foxo4. Conversely, when we suppressed Foxo4 expression using siRNA and examined the Akt activity, we found that reduced Foxo4 expression was associated with a down-regulation of Akt activity (Fig. 5B). Based on these results, it appears that Foxo4 and Akt may positively regulate each other. These findings are in contrast to previous reports, which showed that Akt down-regulated Foxo proteins (29). However, in a recent study it was shown that prolonged Foxo3a activity promoted drug-resistance in leukemia by enhancing the expression of critical signal intermediates that drive the activity of the PI-3 kinase/Akt signaling pathway (31), consistent with our data (Fig. 5A and B). We are currently conducting further experiments to determine how Foxo4 and Akt positively regulate one another. To determine the biological significance of the enhanced level of Foxo4 in the presence of HBX, we suppressed Foxo4 expression using siRNA and subsequently treated Chang-HBX with $\mathrm{H}_{2} \mathrm{O}_{2}$ to induce apoptosis. The Chang-HBX cells treated with Foxo4 siRNA were more susceptible to $\mathrm{H}_{2} \mathrm{O}_{2}$ oxidative stress over the course of treatment compared to Chang-HBX cells treated with the control siRNA (Fig. 5C). Only $40 \%$ of Chang-HBX cells survived at $36 \mathrm{~h}$ after treatment with Foxo4 siRNA under $\mathrm{H}_{2} \mathrm{O}_{2}$-induced oxidative stress, whereas approximately 95\% of Chang-HBX cells survived $36 \mathrm{~h}$ after treatment with control siRNA under $\mathrm{H}_{2} \mathrm{O}_{2}$-induced oxidative stress (Fig. 5C). These results suggest that the Foxo4 transcription factor exerts a protective role against oxidative stress in hepatic cells expressing HBX.

In this study, we have shown that up-regulation of Foxo4 mediated by HBX confers cellular resistance to oxidative stress-induced death. We thus speculate that up-regulation of Foxo4-induced by HBX contributes to the survival of virusinfected liver cells under oxidative stress evoked by HBV replication or chemotherapy and thereby yields a long-term viral reservoir in $\mathrm{HBV}$-infected patients. Moreover, the findings presented in this study suggest that therapeutic strategies selectively disabling the pro-survival actions of Foxo4 may be desirable in HBX-expressing hepatic cells.

\section{Acknowledgements}

This study was supported by a National Research Foundation (NRF) of Korea grant funded by the Ministry of Education, Science and Technology (2009-0071348) and by the World Class University program through NRF funded by the Korean government (R31-2008-000-20004-0). 


\section{References}

1. Neuveut C, Wei Y and Buendia MA: Mechanisms of HBV-related hepatocarcinogenesis. J Hepatol 52: 594-604, 2010.

2. Lupberger J and Hildt E: Hepatitis B virus-induced oncogenesis. World J Gastroenterol 13: 74-81, 2007.

3. Waris G, Huh KW and Siddiqui A: Mitochondrially associated hepatitis B virus X protein constitutively activates transcription factors STAT-3 and NF-kappa B via oxidative stress. Mol Cell Biol 21: 7721-7730, 2001.

4. Kekule AS, Lauer U, Weiss L, Luber B and Hofschneider PH: Hepatitis B virus transactivator $\mathrm{HBx}$ uses a tumour promoter signalling pathway. Nature 361: 742-745, 1993.

5. Lee YH and Yun Y: HBx protein of hepatitis B virus activates Jak1-STAT signaling. J Biol Chem 273: 25510-25515, 1998.

6. Lee YI, Kang-Park S, Do SI and Lee YI: The hepatitis B virus-X protein activates a phosphatidylinositol 3-kinase-dependent survival signaling cascade. J Biol Chem 276: 16969-16977, 2001.

7. Yun C, Cho H, Kim SJ, Lee JH, Park SY, Chan GK and Cho H: Mitotic aberration coupled with centrosome amplification is induced by hepatitis B virus $\mathrm{X}$ oncoprotein via the Ras-mitogen-activated protein/extracellular signal-regulated kinase-mitogen-activated protein pathway. Mol Cancer Res 2: 159-169, 2004.

8. Kim H, Lee YH, Won J and Yun Y: Through induction of juxtaposition and tyrosine kinase activity of Jak1, X-gene product of hepatitis B virus stimulates Ras and the transcriptional activation through AP-1, NF-kappaB, and SRE enhancers. Biochem Biophys Res Commun 286: 886-894, 2001.

9. Chen $\mathbf{J}$ and Siddiqui A: Hepatitis B virus X protein stimulates the mitochondrial translocation of Raf-1 via oxidative stress. J Virol 81: 6757-6760, 2007.

10. van der Horst A and Burgering BM: Stressing the role of Foxo proteins in lifespan and disease. Nat Rev Mol Cell Biol 8: 440-450, 2007

11. Brunet A, Bonni A, Zigmond MJ, Lin MZ, Juo P, Hu LS, Anderson MJ, Arden KC, Blenis J and Greenberg ME: Akt promotes cell survival by phosphorylating and inhibiting a Forkhead transcription factor. Cell 96: 857-868, 1999.

12. Kops GJ, de Ruiter ND, de Vries-Smits AM, Powell DR, Bos JL and Burgering BM: Direct control of the Forkhead transcription factor AFX by protein kinase B. Nature 398: 630-634, 1999.

13. Kops GJ, Dansen TB, Polderman PE, Saarloos I, Wirtz KW, Coffer PJ, Huang TT, Bos JL, Medema RH and Burgering BM: Forkhead transcription factor FOXO3a protects quiescent cells from oxidative stress. Nature 419: 316-321, 2002.

14. Honda Y and Honda S: Oxidative stress and life span determination in the nematode Caenorhabditis elegans. Ann NY Acad Sci 959: 466-474, 2002

15. Furuyama T, Kitayama K, Yamashita $\mathrm{H}$ and Mori N: Forkhead transcription factor FOXO1 (FKHR)-dependent induction of PDK4 gene expression in skeletal muscle during energy deprivation. Biochem J 375: 365-371, 2003.

16. Dijkers PF, Birkenkamp KU,Lam EW, Thomas NS, Lammers JW, Koenderman L and Coffer PJ: FKHR-L1 can act as a critical effector of cell death induced by cytokine withdrawal: protein kinase B-enhanced cell survival through maintenance of mitochondrial integrity. J Cell Biol 156: 531-542, 2002.

17. Dijkers PF, Medema RH, Lammers JW, Koenderman L and Coffer PJ: Expression of the pro-apoptotic Bcl-2 family member Bim is regulated by the forkhead transcription factor FKHR-L1. Curr Biol 10: 1201-1204, 2000.
18. Paik JH, Kollipara R, Chu G, Ji H, Xiao Y, Ding Z, Miao L, Tothova Z, Horner JW, Carrasco DR, Jiang S, Gilliland DG, Chin L, Wong WH, Castrillon DH and DePinho RA: Foxos are lineage-restricted redundant tumor suppressors and regulate endothelial cell homeostasis. Cell 128: 309-323, 2007.

19. Hui RC, Francis RE, Guest SK, Costa JR, Gomes AR, Myatt SS, Brosens JJ and Lam EW: Doxorubicin activates FOXO3a to induce the expression of multidrug resistance gene ABCB1 (MDR1) in K562 leukemic cells. Mol Cancer Ther 7: 670-678, 2008.

20. Yu DY, Moon HB, Son JK, Jeong S, Yu SL, Yoon H, Han YM, Lee CS, Park JS, Lee CH, Hyun BH, Murakami S and Lee KK: Incidence of hepatocellular carcinoma in transgenic mice expressing the hepatitis B virus X-protein. J Hepatol 31: 123-132, 1999.

21. Ford J, Jiang $\mathbf{M}$ and Milner J: Cancer-specific functions of SIRT1 enable human epithelial cancer cell growth and survival. Cancer Res 65: 10457-10463, 2005.

22. Cho IR, Jeong S, Jhun BH, An WG, Lee B, Kwak YT, Lee SH, Jung JU and Chung YH: Activation of non-canonical NF-kappaB pathway mediated by STP-A11, an oncoprotein of Herpesvirus saimiri. Virology 359: 37-45, 2007.

23. Wang JH, Yun C, Kim S, Lee JH, Yoon G, Lee MO and Cho H: Reactive oxygen species modulates the intracellular level of HBx viral oncoprotein. Biochem Biophys Res Commun 310: 32-39, 2003.

24. Gu JM, Lim SO, Oh SJ, Yoon SM, Seong JK and Jung G: HBx modulates iron regulatory protein 1-mediated iron metabolism via reactive oxygen species. Virus Res 133: 167-177, 2008.

25. Essers MA, Weijzen S, de Vries-Smits AM, Saarloos I, de Ruiter ND, Bos JL and Burgering BM: FOXO transcription factor activation by oxidative stress mediated by the small GTPase Ral and JNK. EMBO J 23: 4802-4812, 2004

26. Hui L, Zatloukal K, Scheuch H, Stepniak E and Wagner EF: Proliferation of human HCC cells and chemically induced mouse liver cancers requires JNK1-dependent p21 downregulation. J Clin Invest 118: 3943-3953, 2008.

27. Watanabe T, Nakagawa K, Ohata S, Kitagawa D, Nishitai G, Seo J, Tanemura S, Shimizu N, Kishimoto H, Wada T, Aoki J, Arai H, Iwatsubo T, Mochita M, Watanabe T, Satake M, Ito Y, Matsuyama T, Mak TW, Penninger JM, Nishina H and Katada T: SEK1/MKK4-mediated SAPK/JNK signaling participates in embryonic hepatoblast proliferation via a pathway different from NF-kappaB-induced anti-apoptosis. Dev Biol 250: 332-347, 2002.

28. Chung TW, Lee YC and Kim CH: Hepatitis B viral HBx induces matrix metalloproteinase-9 gene expression through activation of ERK and PI-3K/AKT pathways: involvement of invasive potential. FASEB J 18: 1123-1125, 2004.

29. Burgering BM and Kops GJ: Cell cycle and death control: long live Forkheads. Trends Biochem Sci 27: 352-360, 2002.

30. Huang H, Regan KM, Wang F, Wang D, Smith DI, van Deursen JM and Tindall DJ: Skp2 inhibits FOXO1 in tumor suppression through ubiquitin-mediated degradation. Proc Natl Acad Sci USA 102: 1649-1654, 2005

31. Hui RC, Gomes AR, Constantinidou D, Costa JR, Karadedou CT, Fernandez de Mattos S, Wymann MP, Brosens JJ, Schulze A and Lam EW: The forkhead transcription factor FOXO3a increases phosphoinositide-3 kinase/Akt activity in drug-resistant leukemic cells through induction of PIK3CA expression. Mol Cell Biol 28: 5886-5898, 2008. 